\title{
An analysis of the influence of question design on learners' approaches to number pattern generalisation tasks
}

\author{
Duncan Samson and Marc Schäfer \\ Rhodes University \\ Email: d.samson@sacschool.com and m.schafer@ru.ac.za
}

\begin{abstract}
This paper reports on a study of the extent to which question design affects the solution strategies adopted by children when solving linear number pattern generalisation tasks presented in pictorial and numeric contexts. The research tool comprised a series of 22 pencil-and-paper exercises based on linear generalisation tasks set in both numeric and two-dimensional pictorial contexts. The responses to these linear generalisation questions were classified by means of stage descriptors as well as stage modifiers. The method or strategy adopted was analysed and classified into one of seven categories. In addition, a meta-analysis focused on the formula derived for the $n^{\text {th }}$ term in conjunction with its justification. The results of this study strongly support the notion that question design can play a critical role in influencing learners' choice of strategy and level of attainment when solving pattern generalisation tasks. An understanding of the importance of appropriate question design has direct pedagogical application within the context of the mathematics classroom.
\end{abstract}

\section{Introduction and background}

The connection between mathematics and the notion of pattern is prevalent at all levels of mathematical endeavour. Goldin (2002: 197) describes mathematics as "the systematic description and study of pattern" while Sandefur and Camp (2004: 211) suggest that patterns are "the very essence of mathematics, the language in which it is expressed." Perhaps more generalised and all-encompassing, Steen (1988: 616) broadly defines mathematics as "the science of patterns." Pattern, in a broad sense of the word, is by no means restricted to numeric or pictorial patterns, although this is the usual context of the word for most school Mathematics syllabi.

Working with number patterns or number sequences in the classroom offers valuable opportunities for recognising, describing, extending and creating patterns (Hargreaves, Threlfall, Frobisher \& Shorrocks-Taylor, 1999: 67). It has been suggested that these processes have considerable value as a precursor to formal algebra (English \& Warren, 1998). Searching for patterns is also an important strategy for mathematical problem-solving (Stacey, 1989: 147). Furthermore, in their seminal paper on an organising principle for Mathematics curricula, Cuoco, Goldenberg and Mark (1996) identify the search for pattern as a critical habit of mind.

The study of pattern has become an integral component across all grades of the South African school Mathematics curriculum (Department of Education, 2002; Department of Education, 2003b). In the Intermediate Phase (grades 4-6) the importance of number pattern activities is in "laying the foundation for the study of formal algebra in the Senior Phase while at the same time developing important mathematical thinking skills" (Department of Education, 2003a: 37). Number pattern activities in the Senior Phase (grades 7-9) are essentially an extension of the Intermediate Phase. However, in grades 8 and 9 there is an expectation that learners "use algebra and algebraic processes in their description of these patterns" (Department of Education, 2003a: 39). Within the Further Education and Training (FET) band (grades 10-12) learners will "solve problems related to arithmetic, geometric and other sequences and series" as well as "explore real-life and purely mathematical number patterns and problems which develop the ability to generalise, justify and prove" (Department of Education, 2003b: 12).

There are a variety of different number patterns which fall under the above framework, including: linear or arithmetic sequences, quadratic sequences, power sequences, geometric sequences, and Fibonacci-type sequences. While number patterns can be explored purely numerically namely, in terms of patterns presented as a sequence of numerical symbols - implicit in the requirement that learners be able to "provide explanations and justifications and attempt to prove conjectures" (Department of Education, 2003b: 18) is the condition that at least some of the 


\section{An analysis of the influence of question design on learners' approaches to number pattern generalisation tasks}

pattern questions be set in non-numeric or pictorial contexts.

There are numerous pictorial and practical contexts in which pattern questions can be set, among the most obvious being dot patterns, tiling patterns, matchstick patterns as well as two- and three-dimensional building block patterns. Such pattern tasks usually require some form of generalisation of the pattern, usually in terms of algebraic symbols. It can be argued that setting pattern questions within a pictorial context should allow for greater scope in terms of learner problem-solving strategies, since a pictorial representation can readily be reduced to a purely numeric equivalent, provided the pictorial context has been meaningfully understood. However, although pattern problems presented in a pictorial and/or practical context have the potential to widen the scope of solution strategies for some learners, it can be argued that for others this may well create additional complications. An understanding of how question design of such pattern generalisation tasks is likely to influence the approach adopted by children would greatly assist teachers in terms of their choice of such activities. It is within this context that this paper finds both impetus and import.

\section{Theoretical framework}

While embracing the basic tenets of constructivism, central to the broader study is the fundamental notion that constructivism is a descriptive rather than prescriptive philosophy (Towers \& Davis, 2002: 314). Built onto this philosophy is the firm belief in the use of both language and notation systems/representations as important mediators in the process of knowledge construction - both in terms of their contribution to the organisation of the thinking process itself, as well as the cyclical nature of reflection (Kaput, 1991).

The role of visualisation is also a central focus of the broader study. While generalisation problems presented in a pictorial or practical context have the potential to widen the scope of solution strategies for some learners, it is acknowledged that this may well create additional complications for others (Orton, Orton \& Roper, 1999). The types of generalisation activities considered purposefully in this paper include those presented in pictorial contexts, thus allowing for a possible connection to a referential context that has the potential to aid and enhance the generalisation process. Within the context of the broader study, justification is seen to play a central role in communication of mathematical understanding. Learners' justifications of their generalisations are seen to provide "...a window to view their understanding of the general nature of their rules" (Lannin, 2005: 251).

\section{Methodology and data generation}

This paper focuses on more quantitative aspects within the broader study, which is based on a qualitative investigation framed within an interpretive paradigm. It attempts to interrogate learners' responses to various linear generalisation tasks from both a technical as well as strategic viewpoint. More specifically, the research question under consideration is:

To what extent does question design influence (a) strategy choice, (b) stage progress, (c) contextual connectivity the extent to which the justification of the general term makes reference to the pictorial context - and (d) the diversity of expressions for the general term?

The essential character underpinning the data analysis is the treatment of all responses, particularly those that are unexpected or idiosyncratic, with a genuine interest in understanding their character and origins - a firm conviction that "the constructions of others ... have integrity and sensibility within another's framework" (Confrey, 1990: 108).

A case study methodological strategy was adopted and an appropriate group of research participants was identified - the members of a mixed gender, high ability grade 9 class of 24 learners at an independent school in Grahamstown, South Africa. Over a period of three months, the 24 research participants each completed a series of 22 pencil-and-paper exercises based on linear generalisation tasks set in both numeric and two-

$4 ; 7 ; 10 ; 13 ; \ldots$

Figure 1. Number sequences.

\begin{tabular}{|l|l|l|l|l|l|}
\hline Position & 1 & 2 & 3 & 4 & $\ldots$ \\
\hline Number & 3 & 5 & 7 & 9 & $\ldots$ \\
\hline
\end{tabular}

Figure 2. Tabular form. 


\section{Duncan Samson and Marc Schäfer}

dimensional pictorial contexts. For each pattern, participants were required to provide numerical values for the 'next', $10^{\text {th }}$ and $50^{\text {th }}$ terms (Stages 1 , 2 and 3), as well as a written articulation of their reasoning at each stage. Participants were also asked to provide an algebraic expression for the $n^{\text {th }}$ term (Stage 4), and provide a justification for their expression.

The structure of the six different question design formats was guided by insights gleaned from the literature review (see, for example, Stacey, 1989; Orton, 1997). These six formats are summarised below, where the dependent variable refers to the numerical value of the term itself, while the independent variable refers to the position of the term in the sequence.

- Questions 1-5: A single pictorial term in which the underlying structure is unambiguous. Both dependent and independent variable mentioned in the context of the picture.

- Questions 6-10: Two non-consecutive pictorial terms. Both dependent and independent

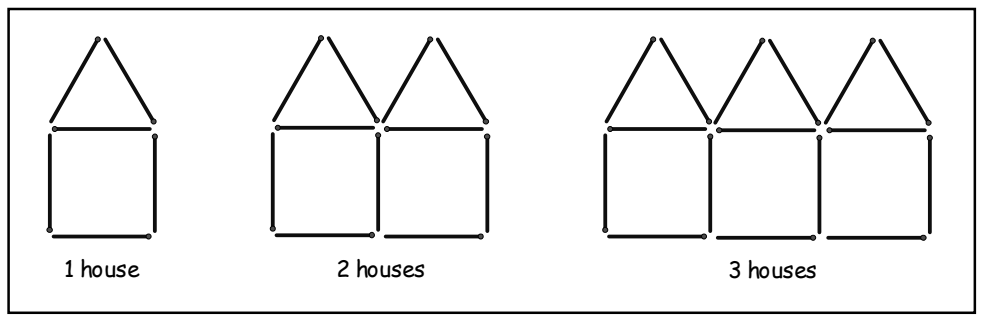

Figure 3. Three consecutive terms variable mentioned in the context of the picture.

- Questions 11-13: Three consecutive purely pictorial terms.

- Questions 14-16: Three consecutive pictorial terms with numerical value of dependent variable indicated.

- Questions 17-19: Three consecutive purely numeric terms (dependent variable indicated).

- Questions 20-22: Three consecutive purely numeric terms in table format (dependent and independent variables indicated).

The responses to the various linear generalisation questions were classified by means of stage descriptors as well as stage modifiers. The method or strategy adopted for determining each of the 'next', $10^{\text {th }}$ and $50^{\text {th }}$ terms was carefully analysed and classified into one of seven categories. In addition, a separate framework was used to characterise each learner's justification of the $n^{\text {th }}$ term in terms of the extent to which the justification was linked to the pictorial context.

Numeric patterns were presented as a simple sequence of numbers (Figure 1) as well as in tabular form (Figure 2). Pictorial patterns were presented using three consecutive terms (Figure 3), two non-consecutive terms (Figure 4) or one single term (Figure 5). The use of single terms was restricted to cases where a single pictorial term provides an unambiguous explanation of the

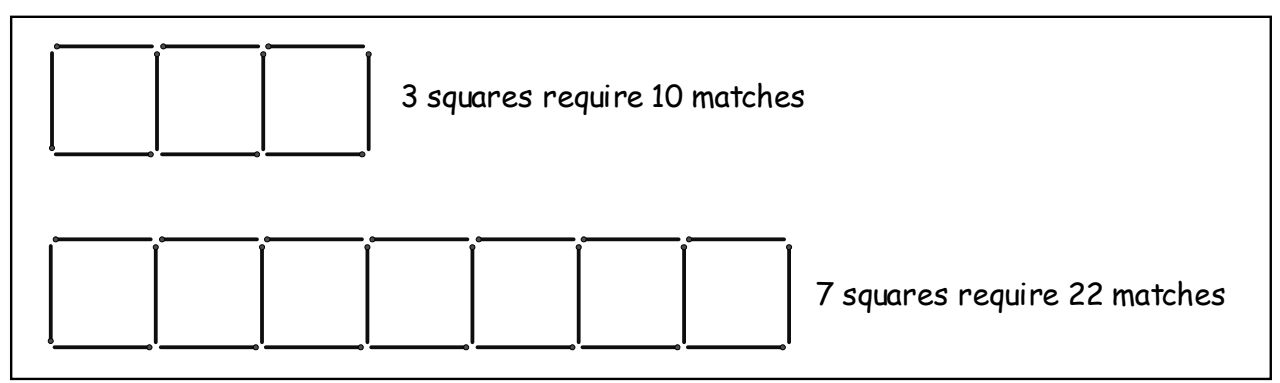

Figure 4. Two non-consecutive terms

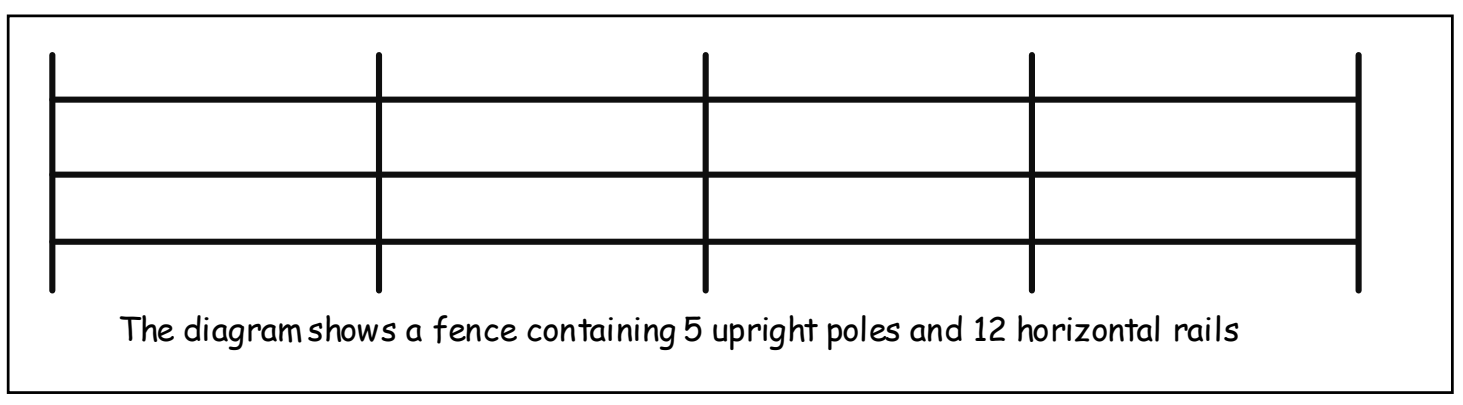

Figure 5. A single pictorial term 


\section{An analysis of the influence of question design on learners' approaches to number pattern generalisation tasks}

underlying structure.

The literature review undertaken to inform the broader study suggested that linear sequences would be most appropriate in terms of eliciting rich data at all levels of the pattern generalisation process. Accordingly, 22 linear/arithmetic sequences of the type $a x \pm c \quad(c \neq 0)$ were chosen. The choice of sequences with non-zero constant terms was a purposeful attempt at ensuring that choice of an inappropriate strategy would not produce a spurious yet numerically correct answer. The 22 sequences were split between pictorial and non-pictorial contexts.

\section{Data analysis}

\section{(a) Stage classification}

For each of the 22 questions, participants were asked to provide numeric values for the 'next', $10^{\text {th }}$ and $50^{\text {th }}$ terms, as well as an algebraic representation for the $\mathrm{n}^{\text {th }}$ term. Using the nomenclature of Stacey (1989: 150), the $10^{\text {th }}$ and $50^{\text {th }}$ terms represent "near generalisation" and "far generalisation" tasks respectively. Determin-ing the $10^{\text {th }}$ term thus represents a task which can be accomplished by means of step-by-step counting or drawing, while determining the $50^{\text {th }}$ term represents a task which goes beyond reasonable practical limits of such a step-by-step approach. The $\mathrm{n}^{\text {th }}$ term denotes an algebraic generalisation of the pattern.

Stage descriptors and stage modifiers were used to classify the responses to the various linear generalisation questions. A similar model was used to that employed by Orton and Orton $(1996 ; 1999)$. The various stage descriptors can be summarised as follows:

- Stage 0: no progress

- Stage 1: 'next' term correctly provided

- Stage 2: 'next' and $10^{\text {th }}$ terms correctly provided

- Stage 3: 'next', $10^{\text {th }}$ and $50^{\text {th }}$ terms correctly provided

- Stage 4: 'next', $10^{\text {th }}, 50^{\text {th }}$ and $\mathrm{n}^{\text {th }}$ terms correctively provided.

The above scheme is not intended as a hierarchical classification system, but rather as a qualitative framework for analysis. Thus, since it is possible for a learner to correctly determine the $50^{\text {th }}$ term despite having incorrectly determined the $10^{\text {th }}$ term (for example), stage modifiers were used to cover all possibilities. The use of both stage descriptors as well as stage modifiers thus allowed for both a quantitative as well as qualitative description of the level of attainment of each participant for each pattern generalisation task.

\section{(b) Strategy classification}

The method or strategy adopted for determining each of the 'next', $10^{\text {th }}$ and $50^{\text {th }}$ terms was carefully analysed and classified into one of seven categories, namely: counting, chunking, difference product, explicit, whole-object uncorrected, wholeobject corrected, and the nature of numerical terms.

The counting method (or method of successive addition) represents a recursive approach whereby subsequent terms are determined by successively adding the identified constant difference to previous terms. The explicit method refers to a strategy where a general formula is first derived for the $\mathrm{n}^{\text {th }}$ term and the desired term is then calculated directly from the general formula by using the independent variable, namely, the position of the term. Provided the general term has been correctly formulated, the explicit method will yield any number of algebraically equivalent expressions for the $\mathrm{n}^{\text {th }}$ term.

\section{(c) Justification classification}

In each question, learners were asked to justify their general formula, that is, to explain why their formula for the $\mathrm{n}^{\text {th }}$ term works. An important aspect of the justification process was an analysis of the extent to which learners used the pictorial scenario as a referential context for the use of a generic example in their justification of the general term. To this end, responses were rated in terms of whether or not the justification was specifically linked to the pictorial - rather than numerical context, using a contextual connectivity rating (CCR). Only those questions that had a pictorial element (Questions 1-16) were rated. Scores of 1, $1 / 2$ or 0 were awarded depending on the extent to which the pictorial context featured in the justification.

\section{Results, analysis and discussion}

Each of the 22 pattern generalisation tasks used in the broader study fell into one of the six different question design formats. Analysis of the influence of question design on (a) strategy choice, (b) stage progress, (c) contextual connectivity, and (d) the diversity of expressions for the general term is discussed in detail below. 


\begin{tabular}{|l|c|c|c|c|}
\hline \multicolumn{1}{|c|}{ Questions } & \multicolumn{4}{|c|}{ Stage } \\
\hline & $\begin{array}{c}\text { Next } \\
\text { (Stage 1) }\end{array}$ & $\begin{array}{c}\mathbf{1 0}^{\text {th }} \\
\text { (Stage 2) }\end{array}$ & $\begin{array}{c}\mathbf{5 0}^{\text {th }} \\
\text { (Stage 3) }^{\text {Stagle }}\end{array}$ & Total \\
\hline $\begin{array}{l}\text { 1-5: Single pictorial term; dependent } \\
\text { and independent variable mentioned. }\end{array}$ & $36.7 \%$ & $2.5 \%$ & $0.0 \%$ & $13.1 \%$ \\
\hline $\begin{array}{l}\text { 6-10: Two non-consecutive pictorial } \\
\text { terms; dependent and independent } \\
\text { variable mentioned. }\end{array}$ & $17.5 \%$ & $0.0 \%$ & $0.0 \%$ & $5.8 \%$ \\
\hline $\begin{array}{l}\text { 11-13: Three consecutive purely } \\
\text { pictorial terms. }\end{array}$ & $38.9 \%$ & $2.8 \%$ & $0.0 \%$ & $13.9 \%$ \\
\hline $\begin{array}{l}\text { 14-16: Three consecutive pictorial } \\
\text { terms; numerical value of dependent } \\
\text { variable indicated. }\end{array}$ & $47.2 \%$ & $2.8 \%$ & $0.0 \%$ & $16.6 \%$ \\
\hline $\begin{array}{l}\text { 17-19: Three consecutive purely } \\
\text { numeric terms; dependent variable } \\
\text { indicated. }\end{array}$ & $51.4 \%$ & $8.3 \%$ & $0.0 \%$ & $19.9 \%$ \\
\hline $\begin{array}{l}\text { 20-22: Three consecutive purely } \\
\text { numeric terms in table format; } \\
\text { dependent and independent variables } \\
\text { indicated. }\end{array}$ & $40.3 \%$ & $1.4 \%$ & $0.0 \%$ & $13.9 \%$ \\
\hline
\end{tabular}

Table 1. Percentage of total responses using counting as sole strategy.

(a) Influence of question design on strategy MacGregor and Stacey (1993) cite one of the main causes of difficulty in formulating algebraic rules as being learners' tendency to focus on the recursive patterns of one variable rather than the relationship linking the two variables. Similar observations have been made by other researchers, for example, Orton (1997). This part of the analysis focuses on the extent to which question design either attracts or discourages a recursive approach.

The counting strategy (recursive approach) was used in one of two different modes, either (a) on its own as sole strategy, or (b) in combination with an explicit strategy. Table 1 shows the percentage of total responses using a counting strategy (as sole strategy) for Stages 1, 2 and 3. The value under the "Total" column indicates the number of responses using the counting strategy as a percentage of the total responses (using any strategy) for Stages 1, 2 and 3 combined. The rationale behind considering only those responses that used counting as the sole strategy was the fact that when counting and explicit strategies were used in combination, the counting strategy was used simply to check the answer derived from the explicit strategy, and was thus not critical to a correct response at that stage.

Table 1 reveals some interesting trends. There is a dramatic drop in the number of learners using the counting strategy when two non-consecutive pictorial terms are used instead of one single pictorial term. There could be two possible reasons for this. Firstly, a single pictorial term may not be a sufficient scaffold to enable some learners to derive a general expression. A second diagram, physically drawn by the learner, may have been necessary in order to see the general structure underlying the pictorial context. Thus, using a counting strategy at Stage 1 may have been a necessary prerequisite to moving to an explicit strategy at Stage 2. Secondly, questions that incorporated two non-consecutive pictorial terms tended to have slightly bigger physical structures compared to the single term scenario, and drawing the next diagram in such a case may have been considered impractical by some learners.

There is a dramatic increase in the number of learners using the counting strategy when three consecutive purely pictorial terms are used instead of two non-consecutive pictorial terms. This increase is even more pronounced when the three consecutive terms are accompanied by an indication of the dependent variable. The initial increase could be a result of two possibilities. Firstly, the fact that the three consecutive pictorial terms are the first three terms in the sequence, the physical structures of the pictorial representations are a little less complex than in the case of the two non-consecutive terms. This may have encouraged learners simply to draw the next term rather than looking for an explicit strategy. Secondly, because the three consecutive terms give a physical 


\section{An analysis of the influence of question design on learners' approaches to number pattern generalisation tasks}

representation of growth, learners may have been drawn to the recursive nature of the pattern and simply added the common difference to the third term in order to obtain a numerical value for the next term. This seemed to be slightly more often the case than simply drawing the next term and counting the number of elements.

The even greater increase when the three consecutive terms are accompanied by an indication of the dependent variable can be explained in terms of the common difference having been made somewhat more explicit by the inclusion of the dependent variable. Learners were thus drawn particularly towards a recursive strategy.

The simple presentation of three consecutive purely numeric terms resulted in the highest proportion of learners opting for the recursive strategy. Just over $51 \%$ of all responses at Stage 1 made use of the counting strategy in the three questions (17-19) presented in this format. Furthermore, just over $8 \%$ of the responses at Stage 2 also made use of the counting strategy, far more than in any other question design. Once again, the common difference becomes immediately clear from the given terms, and learners seem to have been drawn towards this, and used a recursive approach as a result.

Interestingly, when the three consecutive numeric terms are put into table format, which necessarily includes the independent variable, there is a slight drop in the tendency to pattern recursively. One can only surmise that the explicit presence of both dependent and independent variables assisted some learners in seeing a general relation between the two and hence being more inclined to use an explicit strategy over a recursive approach.

The above observations lend support to the findings of Hershkowitz, Dreyfus, Ben-Zvi,

\begin{tabular}{|c|c|}
\hline Questions & Average TSA \\
\hline $1-5$ & 8.91 \\
\hline $6-10$ & 8.98 \\
\hline $11-13$ & 7.97 \\
\hline $14-16$ & 8.68 \\
\hline $17-19$ & 8.75 \\
\hline $20-22$ & 9.32 \\
\hline
\end{tabular}

Table 2. Average TSA per question type.
Friedlander, Hadas and Resnick (2002), that the presentation of consecutive terms encourages recursion, while terms presented non-consecutively tend to encourage generalisation by means of the independent variable. Hershkowitz et al. (ibid.) also found that the use of a pictorial context, particularly if non-consecutive terms were presented, tends to encourage explicit generalisations.

\section{(b) Influence of question design on stage progress}

Table 2 shows the average Total Stage Attainment (TSA) values for each of the six different question designs. The average TSA values are indicative of the level of attainment/progress made by the research participants as a whole. The TSA value was calculated for each individual question by awarding 1 point for a correct Stage 1 response, 2 points for a correct Stage 2 response, 3 points for a correct Stage 3 response, and 4 points for a correct Stage 4 response. The highest obtainable score for a single question is thus $10(1+2+3+4)$ for a learner who correctly answered all four stages.

Although the majority of the average TSA values lie fairly close to one another, of interest are the highest and lowest values, which are well distanced from the rest of the cluster. The highest level of attainment (average TSA = 9.32) was achieved on those questions presented purely numerically, in tabular format. The explicit presence of both the dependent and independent variable, along with the fact that the terms were consecutive and hence made the common difference easier to recognise, all seem to have allowed for greater overall attainment. This finds resonance with a study by English and Warren (1998) where students found it easier to generalise, both verbally and symbolically, when patterns were presented in tabular form as opposed to pictorial form.

The lowest level of attainment (average TSA = 7.97) was achieved on those questions presented as three consecutive purely pictorial terms. In these questions, no mention was made of either the dependent or independent variable. This is an interesting observation when taken in conjunction with the adopted strategy. Question designs that make use of (a) three consecutive purely pictorial terms (Questions 11-13), and (b) three consecutive purely numeric terms in tabular format (Questions 20-22) show almost identical values for the percentage of total responses using an explicit strategy $(85.2 \%$ vs. $84.7 \%)$. However, there is a 


\begin{tabular}{|c|c|}
\hline Questions & Average CCR \\
\hline $1-5$ & 0.86 \\
\hline $6-10$ & 0.80 \\
\hline $11-13$ & 0.64 \\
\hline $14-16$ & 0.40 \\
\hline $17-19$ & - \\
\hline $20-22$ & - \\
\hline
\end{tabular}

Table 3. Average CCR per question type.

marked difference in level of attainment in these two question types ( 7.97 for the former, 9.32 for the latter). This adds weight to the notion that a pictorial representation is only of benefit if the underlying structure can be clearly seen. Despite the fact that learners made almost equal use of an explicit strategy in the two question types, the lower level of success in the purely pictorial context would seem to suggest the use of explicit strategies based on misinterpretation of the general structure inherent in the pictorial context. Thus, while a purely pictorial context may be useful to some learners, to others it may well create complications. A contextualised indication of both the dependent and independent variable (for example: for 2 squares you will need 7 matchsticks), in conjunction with the pictorial representation (Questions 1-5 and 6-10) seemed to be most successful in alleviating this problem.

\section{(c) Influence of question design on contextual connectivity}

Table 3 shows the average Contextual Connectivity Rating (CCR) for each of the four different question designs that were based on a pictorial context (Questions 1-16). The CCR is indicative of the extent to which the justification of the general term makes reference to the pictorial context.

The results shown in Table 3 reveal a fascinating trend. The effect of presenting consecutive terms (Questions 11-13 and 14-16) seems to have a big influence on moving learners' $T_{n}$ justifications away from the referential context (the pictorial representation) toward a more numerically based argument. This effect is even more pronounced in those questions (14-16) where the pictorial context is presented in conjunction with values for the dependent variable. The most

\begin{tabular}{|c|c|}
\hline Questions & $\begin{array}{c}\text { Average number of } \\
\text { correct } T_{n} \text { variations }\end{array}$ \\
\hline $1-5$ & 3.6 \\
\hline $6-10$ & 6.2 \\
\hline $11-13$ & 4.7 \\
\hline $14-16$ & 6.0 \\
\hline $17-19$ & 2.7 \\
\hline $20-22$ & 2.3 \\
\hline
\end{tabular}

Table 4. Average number of $T_{n}$ variations per question type.

likely explanation for this observation is that consecutive terms attract attention to the common difference, hence away from the underlying general structure inherent in the pictorial context, and thus to a more numeric approach to extracting and justifying the general formula for $T_{n}$.

There is also a slight decrease in the average CCR value when moving from questions involving a single pictorial term (Questions 1-5) to those making use of two non-consecutive pictorial terms (Questions 6-10). It is worth keeping in mind that both these question types make contextualised reference to both the dependent and independent variables. Thus, the slight decrease can probably be ascribed to the presence of more numeric points of reference.

\section{(d) Influence of question design on diversity of expressions for $T_{n}$}

Table 4 shows the average number of $T_{n}$ variations per question type. This gives an indication of the diversity of responses in formulating a general algebraic expression for the $\mathrm{n}^{\text {th }}$ term. Only correct Stage 4 (i.e. $T_{n}$ ) responses have been considered.

The dramatic drop in the number of correct $T_{n}$ variations for those questions incorporating purely numeric terms is both expected and understandable, since the lack of a referential (pictorial) context severely limits the scope of readily identifiable variations in $T_{n}$. Without a pictorial frame of reference, expressions for $T_{n}$ can only be derived from purely numeric considerations, the resulting expressions usually taking the form $a+(n-1) d$ or $d n+(a-d)$, or those deriving fortuitously from a guess-and-check approach. 


\section{An analysis of the influence of question design on learners' approaches to number pattern generalisation tasks}

The increase in the number of correct $T_{n}$ variations when moving from questions involving a single pictorial term (Questions 1-5) to those making use of two non-consecutive pictorial terms (Questions 6-10) can probably be ascribed to learners' enhanced appreciation of the underlying general structure inherent in the pictorial context as a result of the additional term. The same argument could be applied when moving from two pictorial terms (Questions 6-10) to three pictorial terms (Questions 11-13 and 14-16). The value of 4.7 (Questions 11-13) is thus somewhat anomalous, and is probably a result of the specific questions chosen for that particular design type. Responses to Stage 4 in Question 13 gave rise to seven different $T_{n}$ variations, while Question 11 and Question 12 had only 4 and 3 respectively. It is worth bearing in mind that some pictorial designs yield fewer accessible (easily identifiable) expressions for $T_{n}$, and this is likely to have been the case in this situation.

Comparing the number of correct $T_{n}$ variations per question type with average CCR values should be treated with extreme caution. There is no reason to assume that a high CCR value implies a high diversity of $T_{n}$ variations. The CCR value relates to the contextualisation of the justification for the $\mathrm{n}^{\text {th }}$ term, but the justification itself is not necessarily an indication of the approach used to derive the algebraic expression for $T_{n}$. It is thus hardly surprising that there is little correlation between the average CCR values per question type and the average number of $T_{n}$ variations per question type.

\section{Conclusion}

This paper is based on a broader study in which a case study approach was adopted as a methodological strategy. Although the emphasis of a case study is to optimise understanding of the specific case under scrutiny rather than generalisation beyond that case, a case study can nonetheless be a useful small step towards a larger generalisation, or an increasingly refined generalisation (Stake, 1994 \& 1995; Cohen \& Manion, 1994). Thus, although any general trends or patterns observed are only relevant to the group of 24 research participants who took part in the study, such "generalisations" could be broadened or increasingly refined by future research involving further samples from the larger population.
Learners' responses gave evidence of the complex interplay between the number pattern itself, the nature of the question design and the specific numeric/pictorial context chosen. Choice of strategy, level of stage progression, contextual connectivity, and the diversity of $T_{n}$ expressions are a manifestation of this interwoven complexity in conjunction with the diverse cognitive skills of each individual learner. There is thus a high degree of interconnectedness, and correlations between different aspects should be treated with due circumspection.

The emphasis of the National Curriculum Statement (NCS) on investigation as a pedagogical approach to number pattern generalisation tasks, as well as its requirement that learners be able to investigate number patterns and hence "make conjectures and generalisations" as well as "provide explanations and justifications and attempt to prove conjectures" (Department of Education, 2003b: 18), has important pedagogical implications for classroom practitioners. An understanding of how question design of such pattern generalisation tasks is likely to influence the approach adopted by children would greatly assist teachers in terms of their choice of such activities. It is within this pedagogical context that this paper finds practical significance.

The results highlighted in this paper give strong support to the notion that question design can play a key role in influencing which strategies are adopted by learners when solving pattern generalisation tasks, in both pictorial and purely numeric contexts. This observation is central to the theme of the broader study, and the notion that different contexts - numeric versus pictorial - will resonate differently with different learners. While a pictorial context may be helpful to some learners, for others it may simply create additional complications.

It would be interesting to repeat this study with other high ability groups of learners, possibly with an augmented selection of patterning questions. This would serve to broaden and/or increasingly refine any localised "generalisations" identified in this paper. In addition, it would add further insight into the complex interplay between the number pattern, the nature of the question design and the specific numeric/pictorial context chosen.

\section{References}

Cohen, L. \& Manion, L. (1994). Research Methods in Education ( $4^{\text {th }}$ Ed.). London: Routledge. 


\section{Duncan Samson and Marc Schäfer}

Confrey, J. (1990). What Constructivism Implies for Teaching. In R. Davis, C. Maher \& N. Noddings (Eds.), Constructivist Views on the Teaching and Learning of Mathematics. Journal for Research in Mathematics Education. Monograph 4 (pp 107-122). Reston, Virginia: NCTM.

Cuoco, A., Goldenberg, E.P. \& Mark, J. (1996). Habits of Mind: An Organizing Principle for Mathematics Curricula. Journal of Mathematical Behaviour, 15, 375-402.

Department of Education (2002). Revised National Curriculum Statement Grades R-9 (Schools): Mathematics. Pretoria: Government Printer.

Department of Education (2003a). Revised National Curriculum Statement Grades R-9 (Schools): Teacher's Guide for the Development of Learning Programmes: Mathematics. Pretoria: Government Printer.

Department of Education (2003b). National Curriculum Statement Grades 10-12 (General): Mathematics. Pretoria: Government Printer.

English, L.D. \& Warren, E.A. (1998). Introducing the Variable through Pattern Exploration. The Mathematics Teacher, 91(2), 166-170.

Goldin, G.A. (2002). Representation in Mathematical Learning and Problem Solving. In L.D. English (Ed.), Handbook of International Research in Mathematics Education (pp 197218). Mahwah, NJ: Erlbaum.

Hargreaves, M., Threlfall, J., Frobisher, L. \& Shorrocks-Taylor, D. (1999). Children's Strategies with Linear and Quadratic Sequences. In A. Orton (Ed.), Pattern in the Teaching and Learning of Mathematics (pp 6783). London: Cassell.

Hershkowitz, R., Dreyfus, T., Ben-Zvi, D., Friedlander, A., Hadas, N. \& Resnick, T. (2002). Mathematics Curriculum Development for Computerised Environments: A DesignerResearcher-Teacher-Learner Activity. In L.D. English (Ed.), Handbook of International Research in Mathematics Education (pp 657694). Mahwah, NJ: Lawrence Erlbaum Associates.
Kaput, J.J. (1991). Notations and Representations as Mediators of Constructive Processes. In E. von Glasersfeld (Ed.), Radical Constructivism in Mathematics Education (pp 53-74). Dordrecht, Netherlands: Kluwer Academic Publishers.

Lannin, J.K. (2005). Generalization and Justification: The Challenge of Introducing Algebraic Reasoning through Patterning Activities. Mathematical Thinking and Learning, 7(3), 231-258.

MacGregor, M. \& Stacey, K. (1993). Seeing a Pattern and Writing a Rule. In I. Hirabayashi (Ed.), Proceedings of the Seventeenth Conference for the Psychology of Mathematics Education (pp 181-188). Tsukuba, Japan.

Orton, J. (1997). Matchsticks, Pattern and Generalisation. Education 3-13, 25(1), 61-65.

Orton, J. \& Orton, A. (1996). Making sense of children's patterning. In L. Puig \& A. Gutiérrez (Eds.), Proceedings of the Twentieth International Conference for the Psychology of Mathematics Education, Volume 4 (pp 83-90). Valencia: Universitat de València.

Orton, J. \& Orton, A. (1999). Pattern and the Approach to Algebra. In A. Orton (Ed.), Pattern in the Teaching and Learning of Mathematics (pp 104-120). London: Cassell.

Orton, J., Orton, A. \& Roper, T. (1999). Pictorial and Practical Contexts and the Perception of Pattern. In A. Orton (Ed.), Pattern in the Teaching and Learning of Mathematics (pp 121-136). London: Cassell.

Sandefur, J. \& Camp, D. (2004). Patterns: Revitalizing Recurring Themes in School Mathematics. Mathematics Teacher, 98(4), 211.

Stacey, K. (1989). Finding and using patterns in linear generalising problems. Educational Studies in Mathematics, 20, 147-164.

Stake, R.E. (1994). Case Studies. In N.K. Denzin \& Y.S. Lincoln (Eds.), Handbook of Qualitative Research (pp 236-247). Thousand Oaks, Calif.: Sage.

Stake, R.E. (1995). The Art of Case Study Research. Thousand Oaks, Calif.: Sage.

Steen, L.A. (1988). The Science of Patterns. Science, New Series, 240, 611-616.

Towers, J. \& Davis, B. (2002). Structuring Occasions. Educational Studies in Mathematics, 49, 313-340.

"The riddle does not exist. If a question can be put at all, then it can also be answered." 